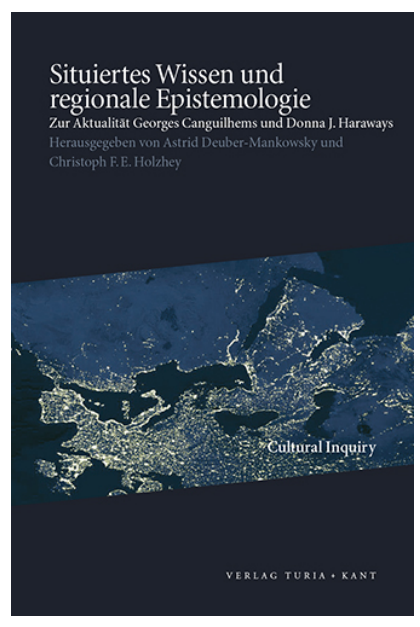

Situiertes Wissen und regionale Epistemologie: Zur Aktualität Georges Canguilhems und Donna J. Haraways, ed. by Astrid Deuber-Mankowsky and Christoph F. E. Holzhey, Cultural Inquiry, 7 (Wien: Turia + Kant, 2013), pp. 223-39

\section{DIDIER DEBAISE}

\section{Nichtmenschliche Subjekte}

\section{Zur Aktualität A.N. Whiteheads}

CITE AS:

Didier Debaise, »Nichtmenschliche Subjekte: Zur Aktualität A.N. Whiteheads «, in Situiertes Wissen und regionale Epistemologie: Zur Aktualität Georges Canguilhems und Donna J. Haraways, hg. v. Astrid Deuber-Mankowsky und Christoph F. E. Holzhey, Cultural Inquiry, 7 (Wien: Turia + Kant, 2013), S. 223-39 <https://doi.org/10.37050/ ci-07_12>

RIGHTS STATEMENT:

(C) by the author(s)

This version is licensed under a Creative Commons AttributionShareAlike 4.0 International License. 


\title{
NICHTMENSCHLICHE SUBJEKTE
}

\author{
Zur Aktualität A.N. Whiteheads \\ Didier Debaise
}

Ausgehend von Alfred North Whiteheads Philosophie möchte ich hier eine spekulative Theorie des Subjektbegriffs vorschlagen. ${ }^{1}$ In den Mittelpunkt dieser spekulativen Theorie müssen wir einen Satz aus Whiteheads Hauptwerk Prozeß und Realität stellen, der besagt, »daß es abgesehen von den Erfahrungen der Subjekte nichts gibt, nichts, nichts, absolutes Nichts «. ${ }^{2}$ Die Hypothese, die ich im Anschluss an diese Aussage verfechten möchte - weil sich in ihr die gesamte Aktualität der Whiteheadschen Philosophie zeigt -, besagt, dass die Frage nach dem Subjekt in seiner Philosophie eine völlig neue Dimension annimmt, indem sie zu einer ontologischen Frage wird: zu einer Frage nach der Natur selbst, unabhängig von einem wahrnehmenden, ausschließlich menschlichen Subjekt. ${ }^{3}$ In diesem Sinne muss es der Anspruch einer spekulativen Theorie des Subjekts im Anschluss an Whitehead sein, folgender Frage einen Sinn geben: Was ist ein nicht-anthropologisches Subjekt?

Beginnen wir mit einer genauen Lektüre des bereits zitierten Satzes: »daß es abgesehen von den Erfahrungen der Subjekte nichts gibt, nichts, nichts, absolutes Nichts «. ${ }^{4}$ Wenn man für einen Augenblick von der repetitiven Struktur absieht, die dieser Aussage einen eigentümlichen Status verleiht, scheint sie auf den ersten Blick einige der grundlegenden Gedanken der Gegenwartsphilosophie nahtlos weiterzuführen. Beschränken wir uns nur auf eine der Hauptbezugsgrößen, die den Raum vorgibt, innerhalb dessen Whitehead seinen eigenen errichtet, nämlich die Philosophie Henri Bergsons. Hat dieser nicht bereits zwei Jahrzehnte vor Whitehead behauptet, dass der sicherste Ausgangspunkt zu einer Erkundung der Natur notwendigerweise bei der Analyse einer privilegierten Perspektive ansetzen müsse - und zwar der unsrigen? Die ersten Sätze der Schöpferischen Entwicklung gehen in diese Richtung:

Das Dasein, dessen wir am gewissesten sind und das wir am besten kennen, ist unleugbar das unsere. Denn von allen übrigen Gegenständen haben wir Eindrücke, die sich als äußerliche und oberflächliche bezeichnen lassen, während wir uns selbst von innen her, in der Tiefe, wahrnehmen. ${ }^{5}$ 
Whitehead selbst hatte einige Jahre zuvor in seinem Buch Der Begriff der Natur ohne offensichtliche Vorbehalte bekräftigt, dass der Begriff der Natur gänzlich auf den Grundlagen einer wahrnehmungsfähigen, menschlichen Erfahrung rekonstruiert werden müsse. Auf die Frage »Was ist die Natur? " schlug Whitehead daher eine Definition vor, die den Naturbegriff auf unsere Wahrnehmung ausrichtet: »Natur ist das, was wir in der Wahrnehmung durch Sinne zur Kenntnis nehmen. In dieser Sinneswahrnehmung wird uns etwas bewußt, was nicht gedacht und gegenüber dem Denken, dem es vorliegt, eigenständig ist. « ${ }^{6}$ In der sinnlichen Wahrnehmung haben wir ein vages Bewusstsein von etwas, das über unser Denken hinausgeht und nicht davon abhängt. Was wir empfinden, ist die Existenz von Ereignissen, die auf mehr oder weniger verschwommene Weise auf die Existenz anderer Ereignisse hinweisen: So nehmen wir etwa neben einem Bruchstück, das auf die Existenz eines Gebäudes hinweist, dessen Teil es ist, in verschwommener Weise auch andere solcher Stücke wahr. Der Gegenstand unserer Wahrnehmung sind »Abschnitte «, »Blöcke «, »Teilstücke«, »Ausschnitte « und partielle Ereignisse, die anderen, mit denen sie verbundenen sind, Zeichen geben. Letztlich handelt es sich um ein komplexes System von Ereignissen, die in unserer unmittelbaren Wahrnehmung angezeigt werden; Ereignisse, die sich sowohl auf die Wahrnehmung beziehen als auch zugleich von ihr unabhängig sind, da sie untereinander direkte Beziehungen unterhalten: »Das unmittelbare Faktum für das Bewußtsein ist nämlich das gesamte Naturgeschehen. «7

Doch weshalb bekräftigt Whitehead einige Jahre später mit einer solchen Insistenz, dass es jenseits der subjektiven Erfahrung nichts gibt? Denn wenn dieser Satz nichts weiter tut, als bereits bestehende Tendenzen der Gegenwartsphilosophie und damit zugleich seiner eigenen Schriften fortzuführen, warum verkündet ihn Whitehead dann so, als ob er gleichsam ein Gabelungspunkt, der Richtungsweiser einer neuen philosophischen Orientierung oder Szene sei? Können wir ihn als schlichte Radikalisierung einer bereits eingeschlagenen Denkweise sehen, in die sich dieser Vorschlag einfach einfügen würde? Wenn man sich an das hält, was wortwörtlich in diesem Satz gesagt wird, kann man diese Vorstellung einer Kontinuität - so scheint es mir - nicht aufrechterhalten. Denn Whitehead begnügt sich nicht etwa damit, den zentralen Ort des Subjekts oder des sinnlichen Bewusstseins in der Erfahrung der Natur zu bekräftigen, sondern geht um vieles weiter, indem er eine Position bezieht, die die reale Existenz der Natur im Allgemeinen im Blick hat. 
Gewiss, sowohl in seinen früheren Arbeiten als auch bei Bergson gibt es eine vergleichbare Ausweitung, »eine Anstrengung [...], über die menschlichen Bedingungen hinauszukommen $" .{ }^{8}$ Auf diese Stelle bezieht sich Deleuze, wenn er in seinem Kommentar bekräftigt: »Seine [Bergsons] Philosophie möchte uns dem Nicht-Menschlichen und dem Übermenschlichen (einer Dauer, die niederer oder höher als die unsere ist ...) öffnen, sie will die Bedingungen, unter denen der Mensch steht, hinter sich lassen [...].«9 Diese Erfahrung des Infra- oder Suprahumanen müsste nun nach Bergson in der einen oder anderen Weise die Zwischensituation des Menschlichen durchschreiten, insofern als das Menschliche der Träger von Dimensionen ist, die es in beide Richtungen hin überschreiten. Wie auch immer das Subjekt aufzufassen sei und welche größeren Dimensionen als das Bewusstseins oder die Intentionalität auch immer miteinbezogen werden mögen - es bleibt die Grundannahme bestehen, dass die einzig legitime Perspektive die unsrige sei und dass die Erforschung der Natur in ihrer Verbindung mit bzw. durch ihre Einschreibung in ein menschliches Subjekt ihren Ausgang nehmen müsse. Wir werden diese Vorstellung, der zufolge ein Satz nur insofern Legitimität und Konsistenz erlangen kann, als er sich - qua Generalisierung oder Analogie - auf seine Einschreibung in ein menschliches Subjekt bezieht, als »anthropologisches Paradigma « bezeichnen. ${ }^{10}$ Demgegenüber ist Whiteheads Satz, wie ich zeigen werde, in direkter Weise ontologisch bzw. seiner Begrifflichkeit gemäß, kosmologisch. Es geht ihm nicht darum zu behaupten, dass wir nicht über unsere eigene Erfahrung als Subjekte hinaus gehen könnten, sondern darum, dass eine Stellungnahme bezüglich der Realität der Natur nicht nur möglich, sondern auch notwendig geworden ist. Die Natur wird nicht mehr als eine Realität gedacht, die einem wahrnehmenden Subjekt gegenübersteht, sondern als Gesamtheit menschlicher und nichtmenschlicher subjektiver Tätigkeiten.

\section{DIE POSSESSIVSUBJEKTE}

Die erste Aufgabe einer Metaphysik des Subjekts besteht also darin, die vermeintlich offenkundige Tatsache zu problematisieren, dass vorrangig Menschen als Subjekte zu bezeichnen sind. Zu diesem Zweck scheint es angebracht, sich des Begriffs zunächst so anzunehmen, dass man sämtliche Kategorien provisorisch einklammert, die ihn in seiner geläufigen Verwendung letzten Endes überlagern und zu seiner Überdeterminie- 
rung geführt haben. Die Frage, die man nun im Rahmen einer metaphysischen Neubegründung zu stellen hätte, wäre, ob es einen Subjektbegriff gibt, der einer Bindung an Kategorien wie Intentionalität, Bewusstsein oder Repräsentation vorgängig ist. Was wären die wichtigsten Komponenten eines solchen Begriffs und wie viele davon gäbe es? Zwar formuliert Whitehead in der Tat das Problem, doch bleibt die Frage bei ihm ohne eindeutige Antwort. Man kann nun einfach versuchen, die heterogenen Linien der konzeptuellen Entwicklungen aufzugreifen, die seine Philosophie durchziehen, um zu sehen, ob sich durch deren Zusammenschluss eine neue Konsistenz des Subjektbegriffs erreichen ließe. Die Anforderungen sind klar: Wir brauchen mindestens eine Komponente des Subjektbegriffs, die in dem Sinne wesentlich zu sein hätte, als dass sie alle Subjekte sowie alle Momente ihrer Existenz betreffen müsste. Und tatsächlich lässt sich in Whiteheads Philosophie ein Begriff finden, der diesem doppelten Anspruch Genüge tut: das Empfinden - feeling. Das Englische verfügt über die im Französischen und Deutschen nicht gegebene Möglichkeit, eine Beziehung zwischen einem Verb in seiner Verlaufsform - to be feeling - und einem Substantiv - to have a feeling - auszudrücken. Whitehead schreibt dies allen Formen subjektiver Erfahrung in der Natur zu, wenn er etwa sagt:

Eine Qualle bewegt sich vorwärts und zieht sich wieder zurück, und indem sie dies tut, verrät sie die Wahrnehmung einer kausalen Beziehung zu der sie transzendierenden Welt; eine Pflanze wächst nach unten, in die feuchte Erde, und nach oben, zum Licht. Es gibt daher einen direkten Grund, hier von dunklen, schwachen Empfindungen eines Kausalnexus zu sprechen, auch wenn wir keinen Anlaß haben, die klaren Wahrnehmungsgegenstände in der Weise der vergegenwärtigenden Unmittelbarkeit zu unterstellen. ${ }^{11}$

Fassen wir diese Erfahrung in ihrer gewöhnlichsten Form auf, dann wird es möglich, ihre ontologischen Dimensionen freizulegen. Was wollen wir bezeichnen, wenn wir beispielsweise sagen, dass ein Tier eine Gefahr verspürt, die seine Umwelt unheimlich werden lässt, oder wenn wir das Gefühl haben, dass sich eine Situation verändern, gefährlich oder angenehm werden könnte? Handelt es sich dabei um dieselbe Erfahrung wie die eines Körpers, der von verschiedenen Sinneseindrücken affiziert ist, die zum Ausdruck bringen, dass er seine Umwelt empfindet? In seiner allgemeinsten Form bezeichnet das Empfinden zugleich die Tatsache, dass die Sinnesdaten der Welt »integriert ", berücksichtigt werden, wie auch den Umstand, dass dies auf eine je eigentümliche 
Weise geschieht. Wenn jene Umgebung unheimlich geworden ist, dann liegt der Grund dafür darin, dass jedes einzelne Gegebene, jedes Datum, das die Umgebung konstituiert, aus der Perspektive einer sich im Werden befindenden Erfahrung zum Teil unheimlich geworden ist. Des Gleichen empfindet der Körper seine Umwelt vermittels von Sinnesorganen, die die Daten in einer eigentümlichen Form, durch einen besonderen Filter hindurch, integrieren: Durch das Auge werden die Dinge gesehen und mit der Hand werden die Tasteindrücke verspürt; ${ }^{12}$ und andere Sinne schließlich entsprechen anderen Formen, die Daten der Welt auf sich auszurichten. ${ }^{13}$

In einem gewissen Sinne lassen alle Erfahrungen in höchst unterschiedlicher Art und Weise etwas erkennen, was als ein Vorgang der Integration oder des Erfassens zu bezeichnen ist, so etwa die Ernährung, das Tastempfinden, das Sehen und auch das Beuteschlagen. Wie auch immer man es bewerten mag: Das Empfinden ist allem voran ein Erfassen, eine eigentümliche Art und Weise des Besitzens, eine Aktivität, bei der etwas ein »Datum aufgreift, um es zu einem Teil [seiner] selbst zu machen. «14 Wir können sogar weiter gehen, indem wir die in den vorangehenden Beispielen implizierten ontologischen Merkmale freilegen und der Behauptung Nachdruck verleihen, dass jedes Erfahrungszentrum aus dem Besitzergreifen, dem possessiven Erfassen unmittelbarer, seine Umwelt bildender Daten besteht und so, nach und nach, das Universum in seiner Gesamtheit ergreift. Was das Tier auf der Lauer empfindet, ist kein spezifisches, die Gefahrengründe bestätigendes Datum, vielmehr empfindet es das gesamte Universum im Modus der Gefahr: Alles wird aussagekräftig. Man wird also zugeben müssen, dass in ontologischer Hinsicht in jedem Empfinden die Gesamtheit des Universums empfunden wird und somit Ästhetik zu Kosmologie wird. Whitehead schließt hier an das Projekt einer Monadologie an, in der die Monaden gleichfalls Zentren von Erfahrungen, d. h. von Perspektiven sind, ihrerseits zusammengesetzt aus allen anderen, oder wie Leibniz schreibt:

Zudem ist jede Substanz wie eine ganze Welt und wie ein Spiegel Gottes oder vielmehr des ganzen Alls, das jede auf ihre Weise ausdrückt, etwa so, wie ein und dieselbe Stadt sich gemäß der verschiedenen Standorte dessen, der sie betrachtet, darstellt. ${ }^{15}$

Auf diese Weise finden sich, so Leibniz weiter, Spuren aller Ereignisse in jedem individuellen Subjekt und "[w]enn man so die Verknüpfung der Dinge recht bedenkt, kann man sagen, daß es zu jeder Zeit in der Seele 
Alexanders [des Großen] Überreste dessen gab, was ihm zugestoßen ist $[\ldots] \ll \cdot{ }^{16}$

Mit der Behauptung, dass das gesamte vorausgehende Universum ohne Ausnahme empfunden wird und dass jedes auf den ersten Blick noch so unbedeutende Ereignis eine Spur hinterlässt, die sich in allen anderen wiederfindet, scheint diese Theorie des Empfindens sehr weit zu gehen. Doch trotz dieser bis dato ungekannten Erweiterung ist sie in Whiteheads Augen noch nicht ausreichend. Merkwürdigerweise engt sie das Empfinden immer noch zu sehr ein. Zu sagen, dass das gesamte Universum einer bestimmten Perspektive gemäß empfunden wird, d. h. also erfasst oder in Besitz genommen wird, reicht nicht aus, weil hier noch eine grundlegende Dimension fehlt - nämlich die Spur all der Möglichkeiten, die ein Empfinden begleiten:

Ein Empfinden trägt die Male seiner Geburt; es erinnert sich in Form eines subjektiven Gefühls an seinen Existenzkampf; es bewahrt den Eindruck von dem, was es hätte sein können, aber nicht ist. [...] Das Wirkliche kann nicht vom Potentiellen geschieden werden und auf bloß Tatsächliches reduziert werden. ${ }^{17}$

Ebendies ist es, was Peirces Pragmatismus als ein »Wäre« (would be), als eine Möglichkeit, bezeichnet. ${ }^{18}$ Das, was hätte sein können, die Wahlen, die vorgenommen wurden, und die Auswahl, die getroffen wurde, definieren ein Subjekt ebenso wie dasjenige, was es gerade ist. Das Empfinden führt all dieses »Wäre« mit sich, all diese Möglichkeiten, die es im Laufe seiner tatsächlichen Existenz ausschlagen musste, und alle Alternativen, die sich ihm boten. Die Tatsache, dass Cäsar den Rubikon nicht hätte überschreiten können und dass ausgehend von der ausgeschlagenen Tat eine ganz andere Welt als diejenige, die wir geerbt haben, hätte existieren können - all dies verleiht der Tatsache, dass er ihn tatsächlich überschritten hat, ihre Bedeutung und singuläre Form. Das Zögern bei der Ausübung einer bestimmten Handlung zeigt, dass andere Möglichkeiten ins Auge gefasst werden, die gleichfalls existentielle Verlaufsbahnen darstellen und zugunsten von einer unter ihnen in der Schwebe gehalten wurden. Auch wenn sie tatsächlich ausgeschlagen werden, macht sie das nicht weniger entscheidend für die tatsächlich ausgeführten Handlungen. So ist jedes positive Empfinden, jedes Erfassen, dauerhaft begleitet von einer ganzen Konstellation von Empfindungen bestehend aus Vermeidungen, Ablehnungen und Zurückweisungen von Möglichem, die ihre Bedeutung wiederum verstärken. Genau dies 
möchte Whitehead sagen, wenn er schreibt: »Das Wirkliche kann nicht vom Potentiellen geschieden werden und auf bloß Tatsächliches reduziert werden. ${ }^{19}$

Indes sollte die Bedeutung dieser Empfindungen von Möglichkeiten, die nicht umgesetzt wurden, nicht überbetont werden. Wenn es so ist, dass die möglichen, gefühlten und mit allen unseren Handlungen und Empfindungen verbundenen Welten konstitutiv für diese Empfindungen sind, wären sie dennoch nur reine Abstraktionen, bar jeder realen Eingeschriebenheit, wenn sie nicht auf direkte Weise mit den ausgeführten, gerade im Vollzug befindlichen Handlungen verbunden und darin verwickelt wären. Die Eventualität, das Zögern angesichts einer Wahlmöglichkeit und die von einer ausgeschlagenen Möglichkeit hinterlassenen Spuren haben Realität nur kraft der tatsächlich ausgeübten Handlungen. Es gibt also in der Erfahrung sehr wohl ein als ontologisch zu bezeichnendes Primat tatsächlicher gegenüber möglichen Empfindungen, d. h. der Handlung gegenüber der Potenz. Dieser Punkt scheint mir grundlegend zu sein, denn er markiert Whiteheads Weigerung, Möglichkeiten losgelöst von realen Handlungen irgendeiner Bewertung zu unterziehen. Das, was ihn interessiert, sind die in Handlungen kristallisierten und in wirklichen Subjekten verkörperten Möglichkeiten. Er führt dafür ein historisches Beispiel an, die Schlacht von Waterloo:

Diese Schlacht führte zum Untergang Napoleons und zu einer Beschaffenheit unserer wirklichen Welt, die auf dieser Niederlage beruht. Aber die abstrakten Begriffe, in denen die Möglichkeiten eines anderen Geschichtsverlaufs zum Ausdruck kommen, der auf seinen Sieg gefolgt wäre, sind relevant für die Tatsachen, die in Wirklichkeit eingetreten sind. Wir denken vielleicht nicht, daß es von praktischer Bedeutung wäre, wenn sich phantasievolle Historiker über solche hypothetischen Alternativen Gedanken machten. Aber wir gestehen ihre Relevanz schon ein, indem wir überhaupt darüber nachdenken, selbst wenn wir sie nur ablehnen. ${ }^{20}$

Je nachdem, wie wir in unsere wirkliche Welt verwickelt sind, sind uns mal mehr, mal weniger intensiv die Möglichkeiten gegeben, die mit einem anderen als jenem durch die Niederlage Napoleons hervorgebrachten Geschichtsverlauf verknüpft sind. Jedes Empfinden in Bezug auf dieses Ereignis ist von der Tatsache gezeichnet, dass es auch nicht hätte stattfinden können, und diese Eventualität schwebt nicht etwa in der Ätherwelt der Abstraktionen, sondern ist nahezu körperlich ins Empfinden eingeschrieben. 
Das Vorhaben einer allgemeinen Theorie der Empfindungen lässt sich in einer mit der Kantischen Philosophie polemisierenden Traditionslinie sehen:

Die organistische [also Whiteheads eigene] Philosophie strebt danach, eine Kritik des reinen Empfindens in der philosophischen Position aufzubauen, in die Kant seine Kritik der reinen Vernunft stellte. Diese sollte auch die übrigen Kritiken verdrängen, die in Kants Philosophie erforderlich sind. Daher wird Kants transzendentale Ästhetik in der organistischen Philosophie zu einem verzerrten Fragment dessen, was sein Hauptthema hätte sein sollen. Das Datum steht in seinen eigenen wechselseitigen Zusammenhängen und die erste Phase des Empfindensprozesses ist die Aufnahme in die entsprechende Übereinstimmung des Empfindens, durch welche das Datum, das eine bloße Potentialität ist, zur individualisierten Grundlage für eine komplexe Einheit der Realisierung wird. ${ }^{21}$

Der Begriff des Empfindens würde demnach zum Schlüsselbegriff einer neuen "Ästhetik «22 und angesichts der Tatsache, dass das Subjekt nichts anderes als eine Vielzahl von Empfindungen ist, käme es damit auch zu einer »ästhetischen « Neudefinition der Subjekte der Natur. Was nun die Neuauflage der Ästhetik angeht, verbleibt Whitehead eher auf der Ebene von Anspielungen. Mir scheint es hingegen möglich zu sein, die im obigen Zitat angeführten Elemente weiter zu denken und sich vorzustellen, wie Whitehead sich zu einem ästhetischen Vorhaben im Sinne Kants und ferner des Großteils seiner geistigen Erben positionieren würde. An erster Stelle stünde der Einwand, dass die Ästhetik weiterhin im Rahmen einer Theorie der Vermögen aufgefasst wird und von denen eines bezeichnet, nämlich »die Fähigkeit (Rezeptivität), Vorstellungen durch die Art, wie wir von Gegenständen affiziert werden, zu bekommen «. ${ }^{23}$ Letzten Endes bezeichnet die Ästhetik die Wahrnehmungsmodi eines Subjekts, die Art und Weise, in der es von den Sinnesdaten affiziert wird. Als Beschränkung erweist sich nun der Umstand, dass man aufgrund dieser Einschreibung der Ästhetik in das Innere eines menschlichen Vermögens Gefahr läuft, die der Natur selbst innewohnenden ästhetischen Dimensionen zu unterschlagen, so als ob die Natur nicht ohnehin schon von einer Vielfalt von Formen des Affiziertwerdens, des Empfindens, des Hoffens oder des Fürchtens erfüllt wäre. ${ }^{24}$ Diese komplexe Operation, durch die die Natur all ihrer ästhetischen Qualitäten entleert wurde oder weniger radikal ausgedrückt: auf ihre ästhetischen Dimensionen hin undurchsichtig gemacht wurde, um diese einem wahrnehmenden Subjekt zuzuschreiben, das sie jenseits seiner selbst projizie- 
ren sollte - diese Operation ist eine der Ausdrucksformen der von Whitehead so genannten »Bifurkation der Natur«:

Eine andere Fassung dieser von mir angegriffenen Theorie besteht in der Bifurkation der Natur in zwei Abteilungen, nämlich in die in das Bewußtsein aufgenommene Natur und diejenige Natur, die die Ursache des Bewußtseins ist. Die Natur, die das in das Bewußtsein aufgenommene Faktum ist, behält das Grünsein der Bäume in sich, den Gesang der Vögel, die Wärme der Sonne und das Gefühl von Samt. Die Natur, die die Ursache des Bewußtseins ist, ist das zusammengereimte System der Moleküle und Elektronen, die den Geist zur Hervorbringung des Bewußtseins der erscheinenden Natur anregen. ${ }^{25}$

Entgegen dieser Bifurkation in eine »wirkliche « und eine »erscheinende Natur " bekräftigt Whitehead, dass »für uns das rote Glühen des Sonnenuntergangs so sehr Teil der Natur sei wie die Moleküle und die elektrischen Wellen «. ${ }^{26}$ " Ästhetik « sollte nicht neben die Art und Weise, Natur zu erleben, gestellt, sondern vielmehr in ihr Inneres, einem Existenzfaktor gleich, zurückverlegt werden. Es ist daher nicht übertrieben zu behaupten, dass die Ästhetik bei Whitehead »ontologisch « wird: Für jedes Sein müsste es eine eigene Ästhetik geben, eine singuläre Weise, durch welche es von der Natur affiziert wird, eine besondere Form des Ausdrucks. Eine solche Theorie der Ausdrucksformen in der Natur hat Whitehead implizit im Blick, wenn er darum bemüht ist, die Ästhetik zu verallgemeinern, sie aus dem Raum der Gegensätze zwischen Natur und wahrnehmendem Subjekt hinaus zu verlagern, um aus ihr den Schlüsselbegriff der Existenz überhaupt von Natur zu machen. Jedes »Datum« ist so bereits von innen her im Zentrum einer Ästhetik, ist bereits von »wechselseitigen Zusammenhängen « und »Übereinstimmungen « mit anderen Erfahrungen beseelt und bereits von Grund auf relational verfasst.

\section{DIE INDIVIDUATION VON SUBJEKTEN}

Doch wie steht es nun genau um die Beziehung zwischen Empfindungen und Subjekt? Wenn die Ästhetik tatsächlich - und mit ihr auch die Frage der Ontologie der Empfindungen - zu einer grundlegenden Angelegenheit wird und sich auf alle Aspekte der Natur erstreckt, bleibt nichtsdestoweniger im einen oder andern Moment ein empfindendes »Subjekt« übrig. Es stellt sich daher die Frage nach dem Vorrang: Ist es 
ein Subjekt, das nunmehr jedes Erfahrungszentrum - und nicht mehr ausschließlich das anthropologische Subjekt - bezeichnet und von dem man sagen kann, das es empfindet, erlebt oder von der Welt affiziert ist, oder aber müssen wir ganz im Gegenteil postulieren, dass die Empfindungen in ursprünglicher Form ohne Subjekt sind? Um dies zu beantworten, unterscheidet Whitehead zwei Bedeutungen des Wortes Subjekt, ${ }^{27}$ die je für eine philosophiegeschichtliche Tradition stehen und die er wieder zu vereinen sucht: ${ }^{28}$ So kann das Subjekt einmal als subjectum, ein andermal im Sinne von superjacio gedacht werden. Beginnen wir mit der ersten Bedeutung: Das Subjekt als subjectum hebt Vorstellungen wie »unterhalb von etwas platziert sein « oder »unter etwas liegen « hervor. Wenn wir eine solche Bedeutung nun an die Frage der Empfindungen zurückbinden, dann können wir sagen, dass das Subjekt gemäß dieser ersten Form als »Träger « oder »Grundlage « von Empfindungen auftritt, eines das zugleich zurückgezogen, im Hintergrund platziert ist und diesen ihren Sinn verleiht. Alles spielt sich entsprechend so ab, als ob das Subjekt voll und ganz im Besitz »seiner « Empfindungen wäre und diese ihm gegenüber sich wie Akzidenzien verhielten, die auf es zukämen, um mal mehr mal weniger stark seine Identität zu beeinträchtigen bzw. seine oberflächlichen Aspekte zum Ausdruck zu bringen. Wenn sich diese Sichtweise eines Subjekts als Besitzer seiner Empfindungen besonders in der neuzeitlichen Philosophie durchgesetzt hat, dann deshalb, weil sich darin in der Tat gewisse grundlegende Züge der Erfahrung niederschlagen. Hierin kommt ein Gefühl zum Ausdruck, wonach sämtliche Erfahrung gleichsam polarisiert und auf ein Subjekt ausgerichtet ist, das in dessen Zentrum steht und von dem aus bestimmte expressive Qualitäten ausstrahlen, wie etwa affektive Grundstimmungen, Klänge, Farben, Tasteindrücke etc. In dem Maße, in dem diese Empfindungen ein Subjekt anzuzeigen scheinen, zu dem sie hintendieren, kann dieses in der Tat als der Träger aufgefasst werden, von dem sie herrühren.

Wie gelingt es nun Whitehead, diese erste Sichtweise des Subjekts in seinem Sinne wieder aufzugreifen? - Indem er ganz einfach die kausale Ordnung verkehrt: Der Eindruck eines Trägers oder eines Fundaments der Empfindungen, wonach es ein Subjekt gäbe, aus dem sich diese abzuleiten scheinen - ein geläufiger und unbestreitbarer Eindruck, dem die Subjektphilosophie eine theoretische Hilfestellung zu geben versuchte - dieser Eindruck ist der Effekt eines Prozesses und nicht sein Ursprung. Er führt hierfür ein Beispiel an: 
Descartes faßt den Denker in seiner eigenen Philosophie als den Schöpfer seiner zufälligen Gedanken auf. Die organistische [d. i. Whiteheads eigene] Philosophie dreht den Spieß um und bestimmt das Denken als eine für die Erschaffung des zufälligen Denkers konstitutive Tätigkeit. Der Denker ist das abschließende Ziel, aufgrund dessen es das Denken gibt. ${ }^{29}$

Die meiste Zeit über verlangt das Denken nichts, außer dass wir es an irgendein Subjekt rückbinden. Wenn wir aber rückblickend versuchen, die Etappen dieser Entwicklung nachzuvollziehen, fügen wir nachträglich ein Subjekt hinzu, das im Grunde genommen davon abgeleitet ist. Daher sollte dieses Subjekt - das voll und ganz im Besitz seiner selbst und per Ableitung auch seiner Empfindungen ist (bzw., wie in Whiteheads Beispiel, seiner Gedanken) und das seinen Affekten, diese gleichsam tragend, zugrunde liegt - nicht als eine Realität ersten Ranges angesehen werden, sondern vielmehr als Abschluss einer »Erfahrungskette «, ${ }^{30}$ als ein Moment, an dem diese voll und ganz sie selbst wird und eine eigene Fülle erlangt. Das Subjekt erweist sich hier als der Moment, in dem die Empfindungen sich in einer einheitlichen Erfahrung kristallisieren, als ein Komplex von Empfindungen, der zu einer singulären Erfahrung geworden ist.

Diese Umkehrung lässt sich sehr gut verallgemeinern und in alle Erfahrungszentren in der Natur verlegen. Ein Tier zum Beispiel ist eine Vielfalt von Erfahrungszentren, welche »die vielen Teile seines Körpers « darstellen, ${ }^{31}$ ihre je eigenen Empfindungen haben und über je eigentümlichen Formen verfügen, affiziert zu werden und Beziehungen mit der ihre eigenen Erfahrungen übersteigenden Umwelt einzugehen. Doch diese vielfältigen Erfahrungszentren, die die Körperteile des Tieres darstellen, sind nicht weniger untereinander verbunden und formen so ein »wirkliches Einzelwesen ", ${ }^{32}$ das diese Vielfalt körperlicher Zentren miteinander kommunizieren lässt und eine komplexe Einheit bildet, die sich als genau dieses empfindende Tier erlebt und manifestiert. Jedes Erfahrungszentrum seines Körpers ist ein Subjekt, in dem Sinne, dass es eine Vielzahl von Empfindungen ausdrückt, die am gleichen Erfahrungspunkt verortet sind; doch bildet die Gesamtheit dieser »Erfahrungszentren ", insofern als diese zu einer höheren Einheit konvergieren, ebenfalls ein Subjekt, welches das Tier als komplexe Einheit von Erfahrungen ist. Eine solche übergeordnete Einheit ist nicht immer erforderlich: »Im Falle von Pflanzen finden wir Körperorganisationen vor, denen es entschieden an diesem Erfahrungszentrum mit höherer Komplexität sowohl von Ausdruck als von angeborenen Daten mangelt. « ${ }^{33}$ Zwar fin- 
den wir hier eine Vielfalt kleiner Erfahrungszentren vor, doch sind sie nicht notwendigerweise einem höheren Zentrum untergeordnet, was Whitehead veranlasst zu sagen:

Eine Pflanze ist eine Demokratie; ein Tier hingegen wird von einem oder mehreren Erfahrungszentren dominiert. Eine solche Dominanz ist allerdings außerordentlich begrenzt. Es ist der Ausdruck des zentralen Lenkers, der sich als ausschlaggebend für die Rezeption der Daten seines Körpers herausstellt. ${ }^{34}$

So gibt diese Auffassung des Subjekts im Sinne von subjectum in der Tat eine wichtige Seite der Erfahrung von Empfindungen wieder, allerdings nur, wenn man darin eine Konsolidierung ${ }^{35}$ dieser Erfahrungen, d. h. den Abschlussmoment eines Prozesses sieht, in dem sich Empfindungen etappenweise zu einer vereinheitlichten Erfahrung verdichten, einer Erfahrung seiner selbst: dieser Körperteil, dieses Tier, dieser Denker.

Doch ist diese Auffassung des Subjekts für sich alleine genommen selbst im Rahmen einer neuen Beziehungslogik der Empfindungen unzulänglich. Sie erlaubt es schließlich nicht, den Einwand zu vermeiden, dass ganz gleich, wie weit man auch immer das Auftauchen des Subjekts verschiebt, sei es an den Anfang oder ans Ende, es doch dabei bleibt, dass irgendwann ein Subjekt konstituiert wird und sich von seinen Empfindungen unterscheidet. Wie ist dieser Teufelskreis zu vermeiden, der von der Tatsache herrührt, dass selbst das auf seine »minimalste « Form reduzierte Subjekt durch nichts erklärt werden kann, das nicht seinerseits bereits subjektiv ist? Genau an dieser Stelle greift Whitehead eine andere Bedeutung des Subjektbegriffs auf, die in einer anderen Traditionslinie eingeschrieben ist, nämlich das Subjekt als Superjekt, abgeleitet von superjacio. Letzteres ließe sich mit einer Reihe von Ausdrücken wie »darüber werfen «, »entgegenwerfen « oder aber »überschreiten «, »überwinden« wiedergeben. Es handelt sich also um ein Subjekt, von dem sich sagen lässt, dass es gewissermaßen seiner selbst voraus ist - in jedem Empfinden virtuell bereits dort ist. Es bezeichnet nicht mehr ein vollständig realisiertes Subjekt, sondern eine Tendenz: »Das Ziel ist jener Gefühlskomplex, in dem diese Daten auf diese Weise erlebt werden. ${ }^{36}$ Alles liegt in der Weise (manière) - der Weise, in der eine Erfahrung gemacht wird; der Weise, in der etwas empfunden wird; der Weise des Erlebens. Dadurch ergibt sich eine Präzisierung der Ästhetik, wie ich es weiter oben beschrieben habe: Jedes Erfahrungszentrum zeichnet sich durch eine eigene Weise aus, durch eine es von allen anderen unterschei- 
dende Klangfarbe. ${ }^{37}$ Es besteht überhaupt kein Bedarf mehr, ein autonomes Subjekt zu postulieren, das im Besitz seiner Erfahrungen wäre, um zu erkennen, dass bereits die Gedanken und sinnlichen Eindrücke - dasjenige, was Whitehead auch eine dem Großteil der Lebewesen gemeinsame viszerale Erfahrung nennt - so viele singuläre Weisen des Verbundenseins mit den Daten umsetzen, wie sie ihnen ihre Umwelten zur Verfügung stellen. Diese Weise ist das Ziel, die Richtung, in die das Empfundene eingebunden oder mobilisiert wird. Man wird daher sagen müssen: »Die Empfindungen sind untrennbar mit dem Ziel verknüpft, auf das sie sich richten; und dieses Ziel ist der Empfindende. Die Empfindungen zielen auf den Empfindenden als ihre Zweckursache. «38

Die beiden Bedeutungen des Subjektbegriffs - subjectum und superjacio - stehen sich demnach nicht entgegen; ganz im Gegenteil können sie in einem erneuerten Denken wiederaufgegriffen werden, in dem Subjekte bar jeder exklusiv anthropologischen Einschreibung sind. Wenn wir nämlich die Frage ausgehend von den Empfindungen stellen, wird offensichtlich, dass es gleichsam zwei Momente eines Empfindens gibt, denen zwei subjektive Phasen entsprechen. Zunächst tendiert das Empfinden in seinem Anfangszustand dazu, sich mit dem, was es empfindet, d. h. mit den Daten, Gefühlen, Vorstellungen und allgemeinen Eindrücken zu vermengen. Doch diese den Daten selbst immanenten Empfindungen sind ihrerseits bereits subjektiv bewohnt. Und in diesem Sinne ist die Weise - die Ausgerichtetheit der Daten - trotz der Tatsache, dass das Empfinden in dieser ersten Phase mit dem Empfundenen nahezu verschwimmt, bereits Ausdruck einer virtuellen Subjektivität (superjacio), ein eigener Empfindungsstil. Erst mit dem Abschluss einer Tätigkeit zeichnet sich eine Erfahrung des Selbst ab, was Whitehead auch als »Genuss des Selbst « (self-enjoyment) bezeichnet - eine Erfahrung des Empfindens als solchem, seines eigenen Stils. So entsteht also ein teilweise ganzes Subjekt (subjectum), das vermittels der Daten, aus denen es hervorgeht, im Besitze seiner selbst ist, wie Gilles Deleuze in seiner Auseinandersetzung mit Whitehead zusammenfasst:

So markiert die Befriedigung, das self-enjoyment, als Schlußphase die Art, in der das Subjekt sich mit sich erfüllt und ein immer reicheres privates Leben erreicht, wenn die Prehension sich mit ihren eigenen Data anfüllt. Das ist eine biblische und auch neuplatonische Auffassung, welche der englische Empirismus (vor allem Samuel Butler) auf den höchsten Punkt getrieben hat. Die Pflanze preist die Ehre Gottes, weil sie sich um so mehr mit sich selbst erfüllt, je mehr sie die Elemente, von denen sie ausgeht, 
intensiv betrachtet und kontrahiert und in dieser Prehension das self-enjoyment ihres eigenen Werden erfährt. ${ }^{39}$

\section{FAZIT: EIN UNIVERSUM VON SUBJEKTIVITÄTEN}

Ich habe hier eine mögliche Version vorgeschlagen, Whiteheads Satz, wonach es »abgesehen von den Erfahrungen der Subjekte [...] nichts, nichts, absolutes Nichts « gebe, philosophisch zu beerben, indem ich ihn auf die Frage nach dem Empfinden als einer Kräftebeziehung zwischen Affizieren und Affiziertwerden und auf die Etablierung einer Ästhetik, die zum Initialmoment einer Kosmologie werden sollte, bezog. Dieser Satz kündigt ein ganzes Programm an, das darauf abzielt, die neuzeitlichen Auffassungen von der Natur neu zu definieren. Nichts verpflichtet uns dazu, unaufhörlich zwischen zwei Naturauffassungen zu oszillieren, die sich in zahlreichen Varianten miteinander verbinden und dennoch mehr oder weniger nahe an ihrer ursprünglichen Form bleiben: Den einen Ansatz nennt Whitehead »romantisch «, den anderen »wissenschaftlich «. Der erste, insbesondere von Shelley und Wordsworth ausdruckstark vertretene, besagt, »daß man die Natur nicht von ihren ästhetischen Werten trennen kann; und daß diese Werte in gewissem Sinne aus der Anhäufung der schwebenden Erscheinung des Ganzen über seinen verschiedenen Teilen hervorgehen $"{ }^{40}$ das heißt aus der nachdrücklichen Präsenz des Universums in jedem einzelnen Fall. Die zweite Auffassung hält dem entgegen: »Die Natur ist eine öde Angelegenheit, tonlos, geruchlos und farblos; nichts als das endlose und bedeutungslose Vorbeihuschen von Material ${ }^{41}{ }^{4}$ dessen ästhetische und axiologische Ausdrucksformen lediglich als »psychische Zutaten « erscheinen, ${ }^{42}$ die durch einen wahrnehmenden Geist einfach hinzugefügt werden. Dieser Gegensatz, ein Erbe der im 17. Jahrhunderten ausgelösten »Bifurkation der Natur", wirkt, ohne auch nur im Geringsten an Kraft eingebüßt zu haben, im gegenwärtigen Denken unaufhörlich fort, und die Gegensätze zwischen Subjekt- und Naturphilosophien scheinen die Bestandteile eines Problems, dessen sie sich nie wirklich entledigen können, lediglich umzuverlagern. Die Subjektivität dringt ins Innere der Natur ein und damit auch die Macht der Empfindungen.

Whiteheads gedankliche Bewegung besteht nun darin, aus diesen »ästhetischen Werten "kein Supplement zu machen, das der Natur durch ein wahrnehmendes Subjekt beigefügt wird, sondern sie als zentrale, ja als wesentlichste Elemente der Natur selbst zu konzipieren. Die 
Ästhetik wird bei Whitehead der Ort jeder Ontologie: Die vielfältigen Weisen des Machens, des Seins und die vielfältigen Fähigkeiten des Affiziertwerdens, in einem Wort: die Modi des »Empfindens « stehen im Mittelpunkt einer Theorie der Natursubjekte. Wir müssen nicht erst den Gegensatz zwischen »Wirklichkeit " und »Wahrnehmung ", "Sein « und "ästhetischem Wert « wiederaufnehmen, um anschließend zu versuchen, sie zu vereinen. Vielmehr kann die Natur direkt als eine Vielfalt von Erfahrungszentren betrachtet werden, die allesamt in direkter Weise expressiv sind. Whiteheads Philosophie ist gewiss eine Kosmologie, und zwar eine, die sich als universaler »Manierismus « charakterisieren lässt. Das Sein und die Weise (manière) unterscheiden sich hier nicht; sie bilden vielmehr die Existenzbedingungen eines jeden Subjekts, sei es menschlich oder nichtmenschlich.

Aus dem Französischen von Konrad Petrovszky

\section{A N MERKUNGEN}

1 In Hinblick auf diese Frage sei auf meine Untersuchung Un empirisme spéculatif. Lecture de Procès et réalité de Whitehead (Paris: Vrin, 2006) verwiesen.

2 Alfred North Whitehead, Prozeß und Realität. Entwurf einer Kosmologie, übers. v. Hans Günter Holl (Frankfurt a.M.: Suhrkamp, 1987), S. 312.

3 Ein solcher Subjektbegriff scheint mir prädestiniert, im Zusammenhang einer Forschung wiederaufgenommen zu werden, die nichtmenschliche Natursubjekte oder das, was ich allgemeiner als »Subjekte der Natur « bezeichnen möchte, zum Gegenstand hat.

4 Whitehead, Prozeß und Realität, S. 312. Diese Aussage blieb bei einem Großteil seiner Leser, die sein geistiges Erbe antraten, über lange Zeit unbemerkt. Ich denke hier in erster Linie an die französische Rezeption seiner Philosophie bei: Henri Bergson, Durée et simultanéité. À propos de la théorie d'Einstein (Paris: Quadrige, 2009), Maurice Merleau-Ponty, Die Natur. Aufzeichnungen von Vorlesungen am Collège de France 1956-1960, hg. v. Dominique Séglard, übers. v. Mira Köller (München: Fink, 2000); Émile Meyerson, Du cheminement de la pensée (Paris: Vrin, 2011); Jean Wahl, Vers le concret. Études d'histoire de la philosophie contemporaine (Paris: Vrin, 1932); Gilles Deleuze, Die Falte. Leibniz und der Barock, übers. v. Ulrich Johannes Schneider (Frankfurt a.M.: Suhrkamp, 1995).

5 Henri Bergson, Schöpferische Entwicklung, übers. v. Gertrud Kantorowicz (Zürich: Coron, 1967), S. 49.

6 Alfred North Whitehead, Der Begriff der Natur, übers. v. Julian von Hassell (Weinheim: VCH, Acta Humaniora, 1990), S. 6.

7 Ebd., S. 15.

8 Henri Bergson, »Einführung in die Metaphysik «, in ders., Denken und schöpferi- 
sches Werden. Aufsätze und Vorträge, übers. v. Leonore Kottje (Hamburg: Europäische Verlagsanstalt, 1993), S. 180-225, hier S. 218.

9 Gilles Deleuze, Henri Bergson zur Einführung, übers. v. Martin Weinmann (Hamburg: Junius, 1997), S. 41.

10 Für eine Analyse des "anthropologischen Paradigmas « in der zeitgenössischen Philosophie siehe die von Quentin Meillassoux entwickelte Idee einer Art des »Korrelationismus «: »Unter /Korrelation« verstehen wir die Idee, derzufolge wir Zugang nur zu einer Korrelation von Denken und Sein haben, und nie gesondert zu einem der beiden Begriffe. Daher nennen wir von jetzt an Korrelationismus jede Denkrichtung, welche den unüberschreitbaren Charakter der so verstandenen Korrelation vertritt."(Quentin Meillassoux, Nach der Endlichkeit [Berlin: Diaphanes, 2008], S. 18)

11 Whitehead, Prozeß und Realität, S. 330.

12 Ebd.

13 Besonders offenkundige Beispiele einer solchen mit den unterschiedlichen Formen sinnlicher Erfahrung zusammenhängenden Vielzahl von Welten finden sich in der Theorie der Umwelt von Uexküll. Vgl. Jakob von Uexküll und Georg Kriszat, Streifzüge durch die Umwelten von Tieren und Menschen. Ein Bilderbuch unsichtbarer Welten (Frankfurt a.M.: Fischer, 1983).

14 Whitehead, Prozeß und Realität, S. 307.

15 Gottfried Wilhelm Leibniz, "Metaphysische Abhandlung «, in ders., Philosophische Schriften, hg. u. übers. v. Hans Heinz Holz, 4 Bde. (Frankfurt a.M.: Suhrkamp, 1996), I: Kleine Schriften zur Metaphysik, S. 49-172, hier S. 77-79.

16 Ebd., S. 77.

17 Whitehead, Prozeß und Realität, S. 414-15.

18 Vgl. Charles Sanders Peirce, "Pragmatism (1907) «, in The Essential Peirce. Selected Philosophical Writings, 2 Bde. (Bloomington: Indiana University Press, 1992), II: 1893-1913 (1998), S. 398-433, hier S. 410-11.

19 Whitehead, Prozeß und Realität, S. 414-15.

20 Ebd., S. 345.

21 Ebd., S. 218.

22 Siehe hierzu die hervorragende Arbeit von Steven Shaviro, Without Criteria. Kant, Whitehead, Deleuze, and Aesthetics (Cambridge: MIT Press, 2009).

23 Immanuel Kant, Kritik der reinen Vernunft, hg. v. Wilhelm Weischedel, 2 Bde. (Frankfurt a.M.: Suhrkamp, 1974), I, S. 69.

24 Der Begriff Ästhetik stammt vom griechischen aisthetikós, das das Vermögen zu empfinden bezeichnet und seinerseits vom Verb aisthánestai abgeleitet ist, das schlicht »empfinden « bedeutet. Die gegenwärtigen Ableitungen des Wortes verbleiben jedoch nicht allein im Rahmen der Ästhetik, sondern verweisen auf alle möglichen Weisen der Blockierung oder Steigerung von Empfindungsvermögen, wie etwa "Anästhetika«. Die Erfindung der modernen, aus den Arbeiten Baumgartens hervorgegangenen Ästhetik, die auf die Frage des "Schönen « und die Wahrnehmung künstlerischer Objekte ausgerichtet war, ist lediglich eine sehr eigentümliche Ausprägung der Geschichte der Ästhetik und es wäre durchaus 
möglich, sich eine Neuauflage des Begriffs vorzustellen, die näher wäre an seiner ursprünglichen Auffassung, sprich eine Theorie der Empfindungen.

25 Whitehead, Begriff der Natur, S. 27.

26 Ebd., S. 25.

27 Für eine ausgiebigere Untersuchung dieser Doppelbedeutung von Subjekt erlaube ich mir auf mein Buch Un empirisme spéculatif zu verweisen.

28 Vgl. dazu Alain de Libera, Archéologie du sujet, Bd. 1: Naissance du sujet (Paris: Vrin, 2007).

29 Whitehead, Prozeß und Realität, S. 283.

30 Zur Frage der Erfahrungsketten und -übergänge siehe William James, Pragmatismus und radikaler Empirismus, hg. u. übers. v. Claus Langbehn (Frankfurt a.M.: Suhrkamp, 2006), darin in erster Linie S. 26-57, »Eine Welt der reinen Erfahrung «. Eine besonders interessante Lektüre dieses Texts legt Bruno Latour vor in »La connaissance est-elle un mode d'existence: rencontre au muséum de James, de Fleck et de Whitehead avec des fossiles de chevaux ", in Vie et expérimentation. Peirce, James, Dewey, hg. v. Didier Debaise (Paris: Vrin, 2007), S. 17-44.

31 Alfred North Whitehead, Denkweisen, hg. u. übers. v. Stascha Rohmer (Frankfurt a.M.: Suhrkamp, 2001), S. 66

32 Ebd.

33 Ebd.

34 Ebd., S. 66-67.

35 Ich beziehe mich an dieser Stelle auf die von Eugène Dupréel entwickelte Theorie der Konsolidierung in "Théorie de la Consolidation ", Revue de l'Institut de Sociologie, 3 (1931), S. 1-58 und 473-530.

36 Whitehead, Denkweisen, S. 182.

37 Dieses Thema scheint mir in erstaunlicher Nähe zur Philosophie von Étienne Souriau zu stehen, in erster Linie zu dem, was er »le sollicitudinaire « nennt. Siehe dazu Étienne Souriau, Avoir une âme. Essai sur les existences virtuelles (Paris: Les belles lettres, 1938) und ders., Les différents modes d'existence (Paris: PUF, 2009) inklusive der hervorragenden von Isabelle Stengers und Bruno Latour verfassten Einleitung.

38 Whitehead, Prozeß und Realität, S. 406.

39 Deleuze, Die Falte, S. 130.

40 Alfred North Whitehead, Wissenschaft und moderne Welt, übers. von Hans Günter Holl (Frankfurt a.M.: Suhrkamp, 1988), S. 107.

41 Ebd., S. 70.

42 Whitehead, Begriff der Natur, S. 35. 
Didier Debaise, »Nichtmenschliche Subjekte: Zur Aktualität A.N. Whiteheads $\ll$, in Situiertes Wissen und regionale Epistemologie: Zur Aktualität Georges Canguilhems und Donna J. Haraways, hg. v. Astrid Deuber-Mankowsky und Christoph F. E. Holzhey, Cultural Inquiry, 7 (Wien: Turia + Kant, 2013), S. 223-39 <https://doi. org/10.37050/ci-07_12>

\section{REFERENCES}

Bergson, Henri, Denken und schöpferisches Werden. Aufsätze und Vorträge, übers. v. Leonore Kottje (Hamburg: Europäische Verlagsanstalt, 1993)

Durée et simultanéité. À propos de la théorie d'Einstein (Paris: Quadrige, 2009)

Schöpferische Entwicklung, übers. v. Gertrud Kantorowicz (Zürich: Coron, 1967)

Debaise, Didier, Un empirisme spéculatif. Lecture de Procès et réalité de Whitehead (Paris: Vrin, 2006)

Debaise, Didier (Hg.), Vie et expérimentation. Peirce, James, Dewey (Paris: Vrin, 2007)

Deleuze, Gilles, Die Falte. Leibniz und der Barock, übers. v. Ulrich Johannes Schneider (Frankfurt a.M.: Suhrkamp, 1995)

_Henri Bergson zur Einführung, übers. v. Martin Weinmann (Hamburg: Junius, 1997)

de Libera, Alain, Archéologie du sujet, Bd. 1: Naissance du sujet (Paris: Vrin, 2007)

Dupréel, Eugène, »Théorie de la Consolidation «, Revue de l'Institut de Sociologie, 3 (1931)

James, William, Pragmatismus und radikaler Empirismus, hg. u. übers. v. Claus Langbehn (Frankfurt a.M.: Suhrkamp, 2006)

Kant, Immanuel, Kritik der reinen Vernunft, hg. v. Wilhelm Weischedel, 2 Bde. (Frankfurt a.M.: Suhrkamp, 1974)

Leibniz, Gottfried Wilhelm, Philosophische Schriften, hg. u. übers. v. Hans Heinz Holz, 4 Bde. (Frankfurt a.M.: Suhrkamp, 1996)

Meillassoux, Quentin, Nach der Endlichkeit (Berlin: Diaphanes, 2008)

Merleau-Ponty, Maurice, Die Natur. Aufzeichnungen von Vorlesungen am Collège de France 19561960, hg. v. Dominique Séglard, übers. v. Mira Köller (München: Fink, 2000)

Meyerson, Émile, Du cheminement de la pensée (Paris: Vrin, 2011)

Peirce, Charles Sanders, The Essential Peirce. Selected Philosophical Writings, 2 Bde. (Bloomington: Indiana University Press, 1992)

Shaviro, Steven, Without Criteria. Kant, Whitehead, Deleuze, and Aesthetics (Cambridge: MIT Press, 2009)

Souriau, Étienne, Avoir une âme. Essai sur les existences virtuelles (Paris: Les belles lettres, 1938)

Les différents modes d'existence (Paris: PUF, 2009) <https://doi.org/10.3917/puf.souri.2009. $01>$

von Uexküll, Jakob u. Georg Kriszat, Streifzüge durch die Umwelten von Tieren und Menschen. Ein Bilderbuch unsichtbarer Welten (Frankfurt a.M.: Fischer, 1983)

Wahl, Jean, Vers le concret. Études d'histoire de la philosophie contemporaine. William James, Whitehead, Gabriel Marcel, 2. erw. Aufl. (Paris: Vrin, 2004)

Whitehead, Alfred North, Der Begriff der Natur, übers. v. Julian von Hassell (Weinheim: VCH, Acta Humaniora, 1990)

—Denkweisen, hg. u. übers. v. Stascha Rohmer (Frankfurt a.M.: Suhrkamp, 2001)

Prozeß und Realität. Entwurf einer Kosmologie, übers. v. Hans Günter Holl (Frankfurt a.M.: Suhrkamp, 1987) 
— Wissenschaft und moderne Welt, übers. von Hans Günter Holl (Frankfurt a.M.: Suhrkamp, 1988) 